\title{
MEROMORPHIC SOLUTIONS OF LINEAR DIFFERENCE EQUATIONS WITH POLYNOMIAL COEFFICIENTS*
}

ABStRaCT. We study the growth of the transcendental meromorphic solution $f(z)$ of the linear difference equation:

$$
\sum_{j=0}^{n} p_{j}(z) f(z+j)=q(z),
$$

where $q(z), p_{0}(z), \ldots, p_{n}(z)(n \geq 1)$ are polynomials such that $p_{0}(z) p_{n}(z) \not \equiv 0$, and obtain some necessary conditions guaranteeing that the order of $f(z)$ satisfies $\sigma(f) \geq 1$ using a difference analogue of the Wiman-Valiron theory. Moreover, we give the form of $f(z)$ with two Borel exceptional values when two of $p_{0}(z)$, $\ldots, p_{n}(z)$ have the maximal degrees.

KEY WORDS: growth, difference equation, Borel exceptional value.

AMS Mathematics Subject Classification: 30D35, 39A10.

\section{Introduction and main results}

Let $f(z)$ be a meromorphic function in the whole complex plane $\mathbb{C}$, we shall use the standard notations of Nevanlinna's theory (see, e.g., $[9,15]$ ), such as the characteristic function $T(r, f)$. Moreover, we will use the notation $S(r, f)$ to denote any quantity that satisfies $S(r, f)=o(1) T(r, f)$ as $r \rightarrow \infty$ outside of a possible exceptional set of finite logarithmic measure. And we will use the notation $\sigma(f)$ to denote the order of growth of $f(z)$ and the notations $\lambda(f)$ and $\lambda(1 / f)$ to denote the exponent of convergence of the zeros and poles of $f(z)$, respectively. We define the difference operators of $f(z)$ by $\Delta f(z)=f(z+1)-f(z)$ and $\Delta^{n} f(z)=\Delta\left(\Delta^{n-1} f(z)\right)=$ $\sum_{i=0}^{n}(-1)^{n-i}\left(\begin{array}{l}n \\ i\end{array}\right) f(z+i)$, where $n(\geq 2)$ is an integer.

* This research was supported by the NNSF of China no. 11171013, 11371225, 11201014 and the Fundamental Research Funds for the Central University. 
In 1935, Whittaker [13] proved that the difference equation $f(z+1)=$ $\psi(z) f(z)$ admits a meromorphic solution of order $\sigma(f) \leq \sigma(\psi)+1$, where $\psi(z)$ is a finite order entire function. In the 1980 s, some mathematicians (see, e.g. $[1,12,14]$ ) obtained more existence theorems about the meromorphic solutions of difference equations. At the beginning of the 21st century, Halburd and Korhonen [6] and Chiang and Feng [3] proved a difference analogue of the logarithmic derivative lemma independently, which provides an efficient tool to study the properties of complex difference equations. By using this new result, Chiang and Feng [3] investigated the growth of meromorphic solutions for higher order linear difference equation

$$
\sum_{j=0}^{n} p_{j}(z) f(z+j)=0,
$$

where $p_{j}(z), j=0, \ldots, n(n \geq 1)$ are entire functions or polynomials. They proved the following two theorems.

Theorem 1 (see [3]). Let $p_{0}(z), \ldots, p_{n}(z)$ be polynomials such that there exists an integer $l, 0 \leq l \leq n$, such that

$$
\operatorname{deg}\left(p_{l}\right)>\max _{0 \leq j \leq n, j \neq l}\left\{\operatorname{deg}\left(p_{j}\right)\right\} .
$$

If $f(z)$ is a meromorphic solution of $(1)$, then $\sigma(f) \geq 1$.

Theorem 2 (see [3]). Let $p_{0}(z), \ldots, p_{n}(z)$ be entire functions such that there exists an integer $l, 0 \leq l \leq n$, such that

$$
\sigma\left(p_{l}\right)>\max _{0 \leq j \leq n, j \neq l}\left\{\sigma\left(p_{j}\right)\right\} .
$$

If $f(z)$ is a meromorphic solution of $(1)$, then $\sigma(f) \geq \sigma\left(p_{l}\right)+1$.

Remark 1. Laine and Yang [10] completed the proof of Theorem 2 by showing that the conclusion of Theorem 2 still holds if there exists an integer $l, 0 \leq l \leq n$ so that among those having the maximal order $\sigma=$ $\max _{0 \leq l \leq n} \sigma\left(p_{l}\right)$, exactly $p_{l}$ has its type strictly greater than the others.

By proving Theorem 1 and Theorem 2, Chiang and Feng [3] have shown that Whittaker's conclusion $\sigma(f) \leq \sigma(\psi)+1$ can be replaced by $\sigma(f)=$ $\sigma(\psi)+1$ (see [3, Corollary 9.3]). Some mathematicians(see, e.g., [2, 4, 8, 11, 16]) then made their efforts to improve Theorem 1 by weakening the conditions. We recall from [4] and [11] the following two results, where $\lambda_{f}$ denotes $\max \{\lambda(f), \lambda(1 / f)\}$ for simplicity. 
Theorem 3 (see [4]). Let $q_{0}(z), \ldots, q_{n}(z)$ be polynomials such that $q_{0}(z) q_{n}(z) \not \equiv 0$ and

$$
\operatorname{deg}\left(q_{0}\right) \geq \max _{1 \leq j \leq n}\left\{\operatorname{deg}\left(q_{j}\right)\right\}
$$

If $f(z)$ is a transcendental meromorphic solution of the following difference equation

$$
\sum_{j=0}^{n} q_{j}(z) \Delta^{j} f(z)=0
$$

then $\sigma(f) \geq 1$.

Theorem 4 (see [11]). Let $q(z), p_{0}(z), \ldots, p_{n}(z)$ be polynomials such that $p_{0}(z) p_{n}(z) \not \equiv 0$ and

$$
\operatorname{deg}\left(\sum_{j=0}^{n} p_{j}(z)\right)=\max _{0 \leq j \leq n}\left\{\operatorname{deg}\left(p_{j}\right)\right\} \geq 1 .
$$

If $f(z)$ is a transcendental meromorphic solution of the following difference equation

$$
\sum_{j=0}^{n} p_{j}(z) f(z+j)=q(z)
$$

then $\sigma(f) \geq 1$. Moreover, if $f(z)$ has finite order, then $1 \leq \sigma(f) \leq 1+\lambda_{f}$.

Theorem 3 improves Theorem 1 because we can use the relation $g(z+l)=$ $\sum_{j=0}^{l}\left(\begin{array}{l}l \\ j\end{array}\right) \Delta^{j} g(z), l=0, \ldots, n$ to rewrite (1) as the form of $(2)$ and it follows that the only coefficient with the maximal degree in Theorem 1 implies $q_{0}(z)$ satisfies the condition of Theorem 3 . Now we use the same relation to rewrite (3) as

$$
\sum_{j=0}^{n} q_{j}(z) \Delta^{j} f(z)=q(z)
$$

where $q(z), q_{0}(z), \ldots, q_{n}(z)$ are polynomials such that $q_{0}(z) q_{n}(z) \not \equiv 0$. We study the growth of transcendental meromorphic solution $f(z)$ of $(4)$ and give two conditions ensuring that $f(z)$ has order of growth no less than 1 . We prove the following Theorem 5 .

Theorem 5. Let $q(z), q_{0}(z), \ldots, q_{n}(z)$ be polynomials such that $q_{0}(z) q_{n}(z)$ $\not \equiv 0$ and

$$
\operatorname{deg}\left(q_{0}\right) \geq \max _{1 \leq j \leq n}\left\{\operatorname{deg}\left(q_{j}\right)\right\}
$$


or

$$
\operatorname{deg}\left(q_{1}\right) \geq \max _{0 \leq j \leq n, j \neq 1}\left\{\operatorname{deg}\left(q_{j}\right)\right\}
$$

If $f(z)$ is a transcendental meromorphic solution of $(4)$, then $\sigma(f) \geq 1$.

From the processing of rewriting (3) to (4), we easily see that $q_{1}(z)$ in (4) corresponds to $\sum_{j=0}^{n} j p_{j}(z)$, where $p_{j}, j=0, \ldots, n$ are the coefficients of (3). Therefore, we have the following corollary from Theorem 5.

Corollary 1. Let $p_{0}(z), \ldots, p_{n}(z)$ be polynomials such that $p_{0}(z) p_{n}(z)$ $\not \equiv 0$ and

$$
\operatorname{deg}\left(\sum_{j=0}^{n} j p_{j}(z)\right)=d=\max _{0 \leq j \leq n}\left\{\operatorname{deg}\left(p_{j}\right)\right\} \geq 1
$$

If $f(z)$ is a transcendental meromorphic solution of $(3)$, then $\sigma(f) \geq 1$.

Example 1. Ishizaki and Yanagihara [7] proved that the following linear difference equation

$$
\left(6 z^{2}+19 z+15\right) \Delta^{3} f(z)+(z+3) \Delta^{2} f(z)-\Delta f(z)-f(z)=0
$$

admits an entire function with order $1 / 3$. This example shows that none of the two conditions in Theorem 5 can be ignored.

In the rest of this paper, we give another result on the growth of transcendental meromorphic solution of (3) and present the form of $f(z)$ which has two Borel exceptional values in the case that two of the coefficients of (3) have the maximal degrees. We prove the following Theorem 6 .

Theorem 6. Let $q(z), p_{0}(z), \ldots, p_{n}(z)$ be polynomials such that $p_{0}(z) p_{n}(z)$ $\not \equiv 0$ and $l$ and $s(0 \leq l, s \leq n)$ be two distinct integers such that $p_{l}$ and $p_{s}$ satisfy

$$
\operatorname{deg}\left(p_{l}\right)=\operatorname{deg}\left(p_{s}\right)>\max _{0 \leq j \leq n, j \neq l, s}\left\{\operatorname{deg}\left(p_{j}\right)\right\} .
$$

If $f(z)$ is a transcendental meromorphic solution of $(3)$, then $\sigma(f) \geq 1$. Moreover, if $f(z)$ is of finite order and has two Borel exceptional values $\alpha$ $(\neq \infty)$ and $\beta(\neq \alpha)$, then we have

(i) if $\beta=\infty$, then $q(z)-\alpha \sum_{j=0}^{n} p_{j}(z) \equiv 0$ and $f(z)=h(z) e^{a z+b}+\alpha$;

(ii) if $\beta \neq \infty$, then $q(z)=\alpha \sum_{j=0}^{n} p_{j}(z) \equiv 0$ and $f(z)=\frac{\beta-\alpha}{1-h(z) e^{a z+b}}+\alpha$, where $a(\neq 0)$ and $b$ are two constants and $h(z)$ is a nonzero rational function.

Example 2. If $f(z)$ is a period 1 function, that is, $h(z)$ is a nonzero constant and $a=2 k i \pi, k \in \mathbb{Z} \backslash\{0\}$, then it is easy to see that $f(z)$ always 
satisfies equation (3) when $q(z) \equiv 0$ and $\sum_{j=0}^{n} p_{j}(z) \equiv 0$. Therefore, both the two cases of Theorem 6 can occur. In the non-periodic case, for example, the function $f(z)=z e^{2 i \pi z}$ has two Borel exceptional values 0 and $\infty$ and satisfies the following difference equation

$$
(z+2) f(z+2)-(z+4) f(z+1)+f(z)=0 .
$$

\section{Some lemmas}

Let $f(z)=\sum_{n=0}^{\infty} a_{n} z^{n}$ be an entire function, we denote the maximum modulus of $f(z)$ on $r>0$ by $M(r, f)=\max _{|z|=r}|f(z)|$ and the central index of $f(z)$ by $\nu(r, f)$, which is defined as the greatest exponent of the maximal term of $f(z)$. The following Lemma 1 obtained recently can be regarded as a difference analogue of the classical Wiman-Valiron theory (see, e.g. [9]).

Lemma 1 (see [5]). Let $f$ be a transcendental entire function of order $\sigma(f)=\sigma<1$, let $0<\varepsilon<\min \{1 / 8,1-\sigma\}$ and $z$ be such that $|z|=r$, where

$$
|f(z)|>M(r, f) \nu(r, f)^{-1 / 8+\varepsilon}
$$

holds. Then for each positive integer $k$, there exists a set $E \subset(1, \infty)$ that has finite logarithmic measure, such that for all $r \notin[0,1] \cup E$,

$$
\frac{\Delta^{k} f(z)}{f(z)}=\left(\frac{\nu(r, f)}{z}\right)^{k}\left(1+R_{k}(z)\right)
$$

where $R_{k}(z)=O\left(\nu(r, f)^{-\kappa+\varepsilon}\right)$ and $\kappa=\min \{1 / 8,1-\sigma\}$.

Lemma 2 (see [9]). If $f(z)$ is an entire function of order $\sigma(f)=\sigma$, then

$$
\sigma=\limsup _{r \rightarrow \infty} \frac{\log \nu(r, f)}{\log r}
$$

Lemma 3 (see [3]). Let $f(z)$ be a meromorphic function with order $\sigma(f)=\sigma<\infty$, and let $\eta$ be a fixed non-zero complex number. Then for each $\varepsilon>0$, we have

$$
T(r, f(z+\eta))=T(r, f)+O\left(r^{\sigma-1+\varepsilon}\right)+O(\log r) .
$$

Lemma 4 (see [9]). Let $f(z)$ be a meromorphic function. Then for all irreducible rational functions in $f$,

$$
R(z, f)=\frac{P(z, f)}{Q(z, f)}=\frac{\sum_{i}^{p} a_{i}(z) f^{i}}{\sum_{j}^{q} b_{j}(z) f^{j}}
$$

such that the meromorphic coefficients $a_{i}(z), b_{j}(z)$ satisfy $T\left(r, a_{i}(z)\right)=$ $S(r, f), i=0,1, \ldots, p$ and $T\left(r, b_{i}(z)\right)=S(r, f), i=0,1, \ldots, q$, we have

$$
T(r, R(z, f))=\max \{p, q\} T(r, f)+S(r, f) .
$$




\section{Proof of Theorem 5}

Proof. On the contrary, we suppose that $\sigma(f)=\sigma<1$. From the proof of Theorem 4 in [11], we know that $f(z)$ has only finitely many poles. Therefore, there exists a rational function $S(z)$ such that $F(z)=f(z)-S(z)$ is transcendental entire. Substituting $f(z)=F(z)+S(z)$ into (4), we get

$$
\sum_{j=0}^{n} q_{j}(z) \Delta^{j} F(z)=Q(z)
$$

where $Q(z)=q(z)-\sum_{j=0}^{n} q_{j}(z) \Delta^{j} S(z)$ is a rational function. Since $F(z)$ is transcendental, we may choose an infinite sequence $z_{k}$ such that $\left|z_{k}\right|=r_{k}$ and $\left|F\left(z_{k}\right)\right|=M\left(r_{k}, F\right)$. Let $0<\varepsilon<\kappa=\min \{1 / 8,1-\sigma\}$. By Lemma 1 , we have

$$
\frac{\Delta^{j} F\left(z_{k}\right)}{F\left(z_{k}\right)}=\left(\frac{\nu\left(r_{k}, F\right)}{z_{k}}\right)^{j}(1+o(1))
$$

holds for all $j=1, \ldots, n$. Dividing $q_{0}(z) F(z)$ on both sides of $(7)$ and substituting (8) into the resulting equation gives

$$
\sum_{j=1}^{n} \frac{q_{j}\left(z_{k}\right)}{q_{0}\left(z_{k}\right)}\left(\frac{\nu\left(r_{k}, F\right)}{z_{k}}\right)^{j}(1+o(1))+1=\frac{Q\left(z_{k}\right)}{q_{0}\left(z_{k}\right) F\left(z_{k}\right)} .
$$

By the condition (5) and the fact that $F(z)$ is transcendental, we have

$$
\frac{Q\left(z_{k}\right)}{q_{0}\left(z_{k}\right) F\left(z_{k}\right)}=o(1), \quad \frac{q_{j}\left(z_{k}\right)}{q_{0}\left(z_{k}\right)}=O(1)
$$

for $j=1, \ldots, n$ as $\left|z_{k}\right|=r_{k} \rightarrow \infty$. Moreover, from Lemma 2, we know that $\frac{\nu\left(r_{k}, F\right)}{r_{k}}=o(1)$ as $\left|z_{k}\right|=r_{k} \rightarrow \infty$. Hence (9) is a contradiction when we let $\left|z_{k}\right|=r_{k} \rightarrow \infty$. This implies that $\sigma(f) \geq 1$ when equation (5) holds.

Consider the case that (6) holds. From the above reasoning we see that $\operatorname{deg}\left(q_{0}\right)<\operatorname{deg}\left(q_{1}\right)$ since we have assumed $\sigma(f)=\sigma<1$. By dividing $q_{1}(z) F(z)$ on both sides of (7) and substituting (8) into the resulting equation, we get

$$
\begin{aligned}
\sum_{j=2}^{n} & \frac{q_{j}\left(z_{k}\right)}{q_{1}\left(z_{k}\right)}\left(\frac{\nu\left(r_{k}, F\right)}{z_{k}}\right)^{j}(1+o(1)) \\
& \quad+\frac{\nu\left(r_{k}, F\right)}{z_{k}}(1+o(1))+\frac{q_{0}(z)}{q_{1}\left(z_{k}\right)}=\frac{Q\left(z_{k}\right)}{q_{1}\left(z_{k}\right) F\left(z_{k}\right)},
\end{aligned}
$$

From (6) and the fact that $F(z)$ is transcendental, we have

$$
\frac{Q\left(z_{k}\right)}{q_{1}\left(z_{k}\right) F\left(z_{k}\right)}=o(1), \quad \frac{q_{j}\left(z_{k}\right)}{q_{1}\left(z_{k}\right)}=O(1), \quad \frac{q_{0}\left(z_{k}\right)}{q_{1}\left(z_{k}\right)}=o(1)
$$


for $j=2, \ldots, n$ as $\left|z_{k}\right|=r_{k} \rightarrow \infty$. Note that $\frac{\nu\left(r_{k}, F\right)}{r_{k}}=o(1)$ as $\left|z_{k}\right|=r_{k} \rightarrow \infty$ by Lemma 2 . These results lead (10) to the following

$$
\frac{\nu\left(r_{k}, F\right)}{r_{k}} \leq K \sum_{j=2}^{n}\left(\frac{\nu\left(r_{k}, F\right)}{r_{k}}\right)^{j} \leq n K\left(\frac{\nu\left(r_{k}, F\right)}{r_{k}}\right)^{2},
$$

where $K$ is some positive value, which implies that $\sigma(f)=\sigma(F) \geq 1$ by Lemma 2, a contradiction to our assumption. So we must have $\sigma(f) \geq 1$ when equation (6) holds. This completes the proof.

\section{Proof of Theorem 6}

Proof. (i) We first prove that $\sigma(f) \geq 1$. Let $a_{l}$ and $a_{s}$ be, respectively, the leading coefficients of $p_{l}(z)$ and $p_{s}(z)$ with degree $d \geq 1$. If $\sigma(f)<1$, then from Theorem 4 and Corollary 1 , we know that $\operatorname{deg}\left(p_{l}(z)+p_{s}(z)\right) \leq$ $d-1$ and $\operatorname{deg}\left(l_{l}(z)+s p_{s}(z)\right) \leq d-1$, which implies that $a_{l}+a_{s}=0$ and $l a_{l}+s a_{s}=0$. It follows that $a_{l}=a_{s}=0$, which contradicts the fact that $p_{l}(z)$ and $p_{s}(z)$ both have the maximal degrees. Hence $\sigma(f) \geq 1$.

(ii) When $f(z)$ has two Borel exceptional values, we discuss the following two cases:

Case 1. $\beta=\infty$. By Hadamard's theory, $f(z)$ assumes the form: $f(z)=$ $h(z) e^{g(z)}+\alpha$, where $g(z)$ is a polynomial with $\operatorname{deg}(g(z))=\sigma(f)=k \geq 1$ and $h(z)$ satisfies $\lambda_{h}=\sigma(h)<\sigma(f)=k$. Substituting this equation into (3) and extracting $e^{g(z)}$ on the left-hand side of the resulting equation gives

$$
e^{g(z)}\left(\sum_{j=0}^{n} p_{j}(z) H(z+j)\right)=q(z)-\alpha \sum_{j=0}^{n} p_{j}(z),
$$

where $H(z+j)=h(z+j) e^{g(z+j)-g(z)}, j=0, \ldots, n$. Denote $g(z)=b_{k} z^{k}+$ $b_{k-1} z^{k-1}+\ldots+b_{0}$, where $b_{k}(\neq 0), \ldots, b_{0}$ are constants. Then we have

$$
g(z+j)-g(z)=b_{k} k j z^{k-1}+g_{j}(z), \quad j=1, \ldots, n,
$$

where $g_{j}(z) \equiv 0$ when $k=1$ or $g_{j}(z)$ are polynomials with degree $\operatorname{deg}\left(g_{j}(z)\right) \leq$ $k-2$ when $k \geq 2$. From Lemma 3, we know that $\sigma(H(z+j))<k$ for $j=0, \ldots, n$. If $q(z)-\alpha \sum_{j=0}^{n} p_{j}(z) \not \equiv 0$, then by Lemma 4 , we get from (11) that $T\left(r, e^{g(z)}\right)=S\left(r, e^{g(z)}\right)$, which is impossible. Therefore, $q(z)-\alpha \sum_{j=0}^{n} p_{j}(z) \equiv 0$ and it follows that

$$
\sum_{j=0}^{n} p_{j}(z) e^{g(z+j)-g(z)} h(z+j)=0 .
$$


If $k \geq 2$, then from the definition of the type $\tau(f)$ (see, e.g., [15]) for an entire function $f(z)$ with order $0<\sigma(f)<\infty$, we easily get $\tau\left(p_{j}(z) e^{g(z+j)-g(z)}\right)=$ $k j\left|b_{k}\right|$ for $j=1, \ldots, n$. Obviously, $k n\left|b_{k}\right|>\ldots>k\left|b_{k}\right|$. However, from Remark 1 we know that $\sigma(h) \geq k$, a contradiction to that $\sigma(h)<k$. Hence $k=1$ and so $\lambda_{h}<\sigma(f)=1$. Note that now $p_{l}(z)$ and $p_{s}(z)$ still have the maximal degrees since $e^{g(z+j)-g(z)}, j=1, \ldots, n$ are all nonzero constants. If $h(z)$ is transcendental, then from the first part, we get $\lambda_{h}=\sigma(h) \geq 1$, a contradiction again. So $h(z)$ must be rational and hence $f(z)$ assumes the form: $f(z)=h(z) e^{a z+b}+\alpha$, where $a(\neq 0)$ and $b$ are two constants and $h(z)$ is a rational function.

Case 2. $\beta \neq \infty$. In this case, $f(z)$ satisfies the equation

$$
\frac{f(z)-\beta}{f(z)-\alpha}=h(z) e^{g(z)}
$$

where $g(z)$ and $h(z)$ are defined as above. It follows that $f(z)=\frac{\beta-\alpha}{1-h(z) e^{g(z)}}$ $+\alpha$ and substituting this equation into (3) yields

$$
\sum_{j=0}^{n} \frac{p_{j}(z)}{1-h(z+j) e^{g(z+j)}}=\frac{q(z)-\alpha \sum_{j=0}^{n} p_{j}(z)}{\beta-\alpha} .
$$

For simplicity, denote the polynomial on the right-hand side of (13) by $A(z)$. By multiplying $\prod_{j=0}^{n}\left(1-H(z+j) e^{g(z)}\right)$ on both sides of $(13)$, we get

$$
B_{1}(z) e^{(n-1) g(z)}+\ldots+B_{n-1}(z) e^{g(z)}+B_{n}=A(z) \prod_{j=0}^{n}\left(1-H(z+j) e^{g(z)}\right)
$$

where $B_{1}, \ldots, B_{n}$ are meromorphic functions with order less than $k$. Moreover,

$$
B_{n-1}(z)=\sum_{j=0}^{n}\left[p_{j}(z)\left(H(z+j)-\sum_{i=0}^{n} H(z+i)\right)\right], \quad B_{n}(z)=\sum_{j=0}^{n} p_{j}(z) .
$$

If $A(z) \not \equiv 0$, then the right-hand side of $(14)$ is a polynomial in $e^{g(z)}$ with coefficients of order less than $k$. By Lemma 4, we get from (14) that $n T\left(r, e^{g(z)}\right) \leq(n-1) T\left(r, e^{g(z)}\right)+S\left(r, e^{g(z)}\right)$, which is impossible. Therefore, $A(z) \equiv 0$. Now we have

$$
B_{1}(z) e^{(n-1) g(z)}+\ldots+B_{n-1}(z) e^{g(z)}+B_{n}=0 .
$$


Since (15) is a polynomial in $e^{g(z)}$ of degree $n$ with coefficients of order less than $k$, we conclude from Lemma 4 that $B_{1}(z) \equiv \ldots \equiv B_{n}(z) \equiv 0$. In particular,

$$
\begin{aligned}
B_{n-1}(z) & =\sum_{j=0}^{n} p_{j}(z) H(z+j)-\left(\sum_{j=0}^{n} p_{j}(z)\right)\left(\sum_{i=0}^{n} H(z+i)\right) \\
& =\sum_{i=0}^{n} p_{j}(z) H(z+j) \equiv 0,
\end{aligned}
$$

which is the equation (12) since $H(z+j)=h(z+j) e^{g(z+j)-g(z)}, j=0, \ldots, n$. This implies that $\sigma(f)=1$ and $h(z)$ is a rational function and so $f(z)$ assumes the form: $f(z)=\frac{\beta-\alpha}{1-h(z) e^{a z+b}}+\alpha$, where $a(\neq 0)$ and $b$ are two constants and $h(z)$ is a rational function. This completes the proof.

\section{References}

[1] Bank S.B., Kaufman R.P., An extension of Hölder's theorem concerning the gamma function, Funkcial. Ekvac., 19(1976), 53-63.

[2] Chen Z.X, Growth and zeros of meromorphic solution of some linear difference equations, J. Math. Anal. Appl., 373(2011), 235-241.

[3] Chiang Y.K, Feng S.J., On the Nevanlinna characteristic of $f(z+\eta)$ and difference equations in the complex plane, Ramanujan J., 16(2008), 105-129.

[4] Chiang Y.K, Feng S.J., On the growth of logarithmic differences, difference quotients and logarithmic derivatives of meromorphic functions, Trans. Amer. Math. Soc., 361(2009), 3767-3791.

[5] Chiang Y.K, Feng S.J., On the growth of logarithmic difference of meromorphic functions and a Wiman-Valiron estimate, Constr. Approx., 44(2016), 313-326.

[6] Halburd R.G., Korhonen R.J., Difference analogue of the lemma on the logarithmic derivative with applications to difference equations, J. Math. Anal. Appl., 314(2006), 477-487.

[7] Ishizaki K., Yanaginara N., Wiman-Valiron method for difference equations, Nagoya Math. J., 175(2004), 75-102.

[8] Ishizaki K., On difference Riccati equations and second order linear difference equations, Aequat. Math., 81(2011), 185-198.

[9] Laine I., Nevanlinna theory and complex differential equations, De Gruyter Studies in Mathematics, vol. 15, Walter de Gruyter \& Co., Berlin, 1993.

[10] Laine I., Yang C.C., Clunie theorems for difference and $q$-difference polynomials, J. Lond. Math. Soc., 76(2)(2007), 556-566.

[11] Li S., GaO Z.S., Finite order meromorphic solutions of linear difference equations, Proc. Japan Acad. Ser. A Math. Sci., 87(2011), 73-76.

[12] Shimomura S., Entire solutions of a polynomial difference equation, J. Fac. Sci. Univ. Tokyo Sect. IA Math., 28(1981), 253-266. 
[13] Whittaker J.M., Interpolatory Function Theory, Cambridge Tracts in Mathematics and Mathematical Physics, No. 33, Stechert-Hafner, Inc., New York, 1964.

[14] Yanagihara N., Meromorphic solutions of some difference equations, Funkcial. Ekvac., 23(1980), 309-326.

[15] Yang C.C., Yi H.X., Uniqueness Theory of Meromorphic Functions, Mathematics and its Applications, vol. 557, Kluwer Academic Publishers Group, Dordrecht, 2003.

[16] Zheng X.M., Tu J., Growth of meromorphic solutions of linear difference equations, J. Math. Anal. Appl., 384(2011), 349-356.

YUEYANG ZHANG

LMIB \& School of MATHEMATicA

And Systems Science

BEIHANG UNIVERSITY

Beijing, 100191, P.R. China

e-mail: zyynszbd@163.com

ZongSheng GaO

LMIB \& School of Mathematica

And Systems ScIEnCE

BEIHANG UNIVERSITY

Beijing, 100191, P.R. China

e-mail: zshgao@buaa.edu.cn

HUILIANG ZHANG

LMIB \& School of Mathematica

AND Systems SCIENCE

BEIHANG UNIVERSITY

BeiJing, 100191, P.R. ChinA

e-mail: 205191566@qq.com

Received on 20.10.2016 and, in revised form, on 08.11.201\%. 